\title{
NARRATIVAS TRANSMÍDIA E GAMIFICAÇÃO: CRIAÇÃO DE NOVOS CENÁRIOS EDUCATIVOS ATRAVÉS DA INTERMEDIAÇÃO TECNOLÓGICA NO ENSINO MÉDIO
}

\author{
SALVADOR/BA JULHO/2018
}

\author{
Sandra Lúcia Pita de Oliveira Pereira \\ - UFBA/CEMITEC - sandrapita@uol.com.br \\ Graça Regina Armond Matias Ferreira \\ - UFBA/CEMITEC - ginamatias@hotmail.com
}

Tipo: Investigação Científica (IC)

Natureza: Planejamento de Pesquisa

Categoria: Métodos e Tecnologias

Setor Educacional: EDUCAÇÃO MÉDIA E TECNOLÓGICA

\begin{abstract}
RESUMO
Este projeto surge da necessidade de estudos sobre narrativas transmídia no processo de gamificação de aulas no Ensino Médio. Constituindo uma primeira reflexão baseada na revisão da literatura, proponho definir o conceito, descrever algumas experiências que ajudam à sua compreensão e mostrar as potencialidades desta abordagem para o desenvolvimento de novos cenários de ensino e aprendizagem. O objetivo é compreender de que maneira poderão as narrativas transmídia contribuir para a inovação das práticas pedagógicas dos professores e para a transformação da realidade escolar com o intuito de tornar a escola num espaço mais significativo, inovador e empreendedor. Esta técnica de contar e mergulhar em histórias com recurso nomeadamente às ferramentas Web 2.0 já está a ser amplamente utilizada para filmes, televisão e publicidade, começando também a interessar e a ser estudada no âmbito educacional dadas as suas potencialidades para a inovação na educação. Através do desenvolvimento desta pesquisa, o aluno do campo, terá oportunidade de, através das vivências de investigação atreladas ao currículo de ciências e ao uso das tecnologias, participar de atividades pedagógicas que aliem o conhecimento prévio com as propostas experimentais; para que alunos e professores da educação básica possam imergir na iniciação científica e instigar a vontade de aprender e pesquisar de maneira natural dentro do espaço da sala de aula, potencializando o ciberespaço como componentes pedagógicos atrelados às aulas gamificadas relacionadas aos conteúdos de Ciências das Natureza, em seu ambiente natural - as comunidades do Emitec e de forma híbrida no Colégio Montessoriano.
\end{abstract}

Palavras-chave: Educação. Gamificação. Narrativas transmídia. Práticas pedagógicas. Web 2.0.

AGRADECIMENTOS

MARIA LUCIA MATOS E JURACI MATOS, PELO APOIO INCONDICIONAL AOS TRABALHOS DESENVOLVIDOS NO COLÉGIO MONTESSORIANO. 


\section{Introdução}

O aumento do uso de tecnologias pelos alunos fora da escola e as oportunidades de aprendizagem informal que aí têm, cria um novo impulso para uma aprendizagem mais aberta às tecnologias dentro da própria escola. A discussão e a criação de uma aprendizagem mais personalizada, o uso e a maior familiaridade dos educadores com essas tecnologias poderá permitir que muitos professores repensem as suas práticas pedagógicas, considerando a possibilidade de vir a usar essas tecnologias em sala de aula. As narrativas transmídia, entendidas como "novos cenários educativos" são uma área nova que pode oferecer um grande potencial em termos de inovação dos processos de organização do ensino e da aprendizagem.

\section{Narrativas Transmídia}

O conceito de narrativas transmídia está associado a várias definições (Scolari, 2009). O termo transmídia surgiu pela primeira vez em 1993, quando Marsha Kinder publica o seu livro Playing with Power in Movies, television, and video games: from Muppet Babies to Teenage Mutant Ninja Turtles, Marsha Kinder e cria o conceito de "transmídia intertextual" para definir um supersistema de entretenimento que promove uma intertextualidade transmídia, isto é, uma mistura criteriosa de animais, ficção científica, artes marciais e de difusão em vários meios de comunicação.

No entanto, foi em 2003, que Henry Jenkins cunhou a expressão narrativas transmídia transmedia storytelling num artigo publicado pela Technology Review. Segundo o autor "na forma ideal de narrativa transmídia, cada mídia faz o que faz de melhor - de forma que a história possa ser introduzida num filme, expandida através da televisão, novelas, gamificação e que o seu mundo possa ser explorado e experienciado durante um jogo (Jenkins (2003, p.3). Partindo do princípio de que as pessoas aprendem de diversas maneiras e que contar histórias é uma tradição da humanidade, na prática, o que Jenkins propõe é que faz sentido contar histórias usando uma variedade de meios de comunicação, isto é, na forma de narrativas transmídia (Jenkins, 2009). O que significa que ser transmitido, por exemplo, em gamificação, não é a mesma coisa que ser transmitido na televisão ou no cinema. Todos os tipos de mídia contribuem para a construção da narrativa e é esta disseminação textual que se torna importante na cultura popular do nosso cotidiano. Robert Pratten (2011) reforça o conceito dizendo que as narrativas transmídia são uma forma de contar uma história através de múltiplos meios de comunicação e, de preferência, embora isso possa não acontecer, com um grau de interação, participação e colaboração da audiência. Nas narrativas transmídia, o envolvimento com cada mídia aumenta a compreensão do autor, o prazer e afeto pela 
história. Para Gambarato não se trata apenas de oferecer o mesmo conteúdo em diferentes plataformas de mídia, mas sim a construção de uma experiência, desdobrando conteúdo e gerando possibilidades para a história evoluir com conteúdos novos e relevantes (Gambarato, 2012, p.4).

\section{Narrativas Transmídia em Contexto Educativo}

A sociedade está rodeada por um mundo de conteúdos, produtos e oportunidades de entretenimento.

Contar histórias através de diferentes meios de comunicação, permite que o conteúdo tenha o tamanho certo, na hora certa e colocado no sítio certo para formar uma maior, mais coesa e gratificante experiência. Na prática, as narrativas transmídia acabam por não ser um conceito novo mas sim uma nova terminologia para o conceito de comunicação de ideias em diferentes meios de comunicação. Transmídia é o ato de ter um conceito central e transmiti-lo ao consumidor através de múltiplas plataformas, incluindo as tecnologias digitais atuais tais como, mas não só, filmes, jogos de vídeo, programas de televisão, Web 2.0 e celulares em combinação com os meios de comunicação tradicionais, como gamificação, brinquedos, impressões, etc.

Enquanto a transmídia é usada principalmente em indústrias de publicidade, entretenimento e orientada para o utilizador, Bottone (2012) explora as potencialidades das narrativas transmídia na educação e na formação.

As narrativas transmídia têm como objetivo reforçar a ideia central de uma história, com uma variedade de dispositivos e plataformas que fornecem informações adicionais, dão maior importância às personagens secundários na narrativa principal, ou até mesmo adicionando novas personagens à história original. Esta técnica de uso de múltiplas plataformas já está a ser amplamente utilizada em filmes, televisão e publicidade e pode oferecer vantagens significativas para a educação. A potencialidade de trabalhar com narrativas transmídia reside na possibilidade de articular os conteúdos pedagógicos com atividades que já estão presentes no cotidiano dos estudantes, tal como o trabalho colaborativo, a partilha de informações e a interação. O uso de plataformas como sites, blogs, televisão, Facebook, Twitter, Youtube e de dispositivos como o celular, computador e tablet podem auxiliar os educadores no desenvolvimento de estratégias metodológicas capazes de melhor atender às necessidades dos alunos, justamente por se adaptarem ao seu contexto, promoverem a mobilidade, permitirem a produção de conteúdo e a escrita do mesmo em sítios comuns, de serem plataformas de maior difusão e de considerarem também os vários estágios de aprendizagem dos estudantes, 
as suas particularidades e interesses (Porto-renó, Versuti, Moraes Gonçalves \& Gosciola, 2011; Sharda, Dakich \& Smeda, 2012).

Para Illera e Castells (2012), a utilização da narrativa digital - digital storytelling é uma forma de integrar os alunos nas atividades escolares de modo a serem mais ativos e participativos do que o habitual. A narrativa digital é vista como uma ferramenta e não como uma finalidade, isto é, o que se pretende do ponto de vista educativo é permitir construir um significado através de diferentes formas, meios e suportes. Este paralelismo entre os professores utilizando múltiplos meios como o livro, o quadro, o lápis e a narrativa digital tem um interesse pedagógico que é importante a exploração. Do ponto de vista educativo, o que interessa explorar são os aspectos da narrativa transmídia no que concerne à concepção das próprias mensagens multimídia. A transmídia pode alcançar uma população de estudantes que se sente desencantada, negligenciada e invisível, dando-lhes a oportunidade para deixarem a sua marca no mundo e de se fazerem ouvir (Gomez, 2011).

Fleming (2011) afirma que o seu dia a dia se baseia em contar histórias e que o que observa é que existe um grande distanciamento entre os adolescentes e a leitura. Foram as novas tecnologias e a aproximação que os alunos têm com elas que o levaram a repensar o conceito de contar histórias para as crianças do século XXI. Depois de muitas experiências nas suas aulas, a autora propõe um novo modelo de contar histórias com mais sucesso na captação da atenção dos seus alunos: as narrativas transmídia. Para a autora, as narrativas transmídia constituem um novo modelo de narrativa em que as partes mais importantes da história são contadas através de múltiplos espaços onde os leitores têm de se assumir como participantes ativos.

Geoffrey Long (2009) refere, a propósito, o conceito de "educação transmídia" estabelecendo precisamente uma relação entre as narrativas transmídia no entretenimento e as narrativas transmídia na educação. Explica que da mesma forma que a narrativa transmídia através de filmes ou jogos dá aos consumidores a motivação para se procurar saber mais sobre a história, também na educação, os professores podem usar a mesma técnica com os seus alunos de modo a provocar-lhes o desejo de se envolverem mais nos conteúdos em aulas, utilizando diferentes tipos de suporte. Ao utilizar diferentes plataformas para exploração de uma mesma história, como por exemplo, os filmes, jogos e animações, o professor pode criar com os seus alunos uma comunidade de conhecimento, onde cada aluno utiliza o meio de comunicação com o qual mais se identifica e, todos juntos constroem uma grande história, ligando entre si todas as plataformas e dispositivos utilizados. Este tipo de técnica permite que os alunos aprendam a trabalhar em equipe e para um todo, desperta-lhes o desejo e a motivação 
para a construção de conhecimento num mundo ficcional e mostra-lhes também o mundo real, o mundo da comunicação digital, da publicidade e do entretenimento orientado para o consumidor. A narrativa transmídia contribui desta forma, para uma aprendizagem através da participação em comunidades de conhecimento onde os alunos, através da criação de histórias vão desenvolver a sua imaginação. Com esta estratégia aliada a gamificação, as salas de aula, oferecem aos estudantes a oportunidade de se educarem uns aos outros em diferentes tipos de mídia, proporcionando-Ihes um espaço de partilha, de trabalho colaborativo, de pesquisa e um ambiente diferente e, de certa maneira, associado ao mundo real do trabalho (Long, 2009).

Charmaine $\mathrm{Ng}$ (2012) questionando-se sobre como é que os professores implementam as estratégias de transmídia nas suas salas de aula e como é que tomam decisões, refere a necessidade da integração da história no currículo e de elementos transmídia, tais como cartas escritas, animações de vídeo, episódios autónomos e interatividade. Refere que um projeto transmídia deve ser pensado desde o início como uma história que se desenrola ao longo do tempo e em múltiplas plataformas. Sugere, no entanto, que se deve ter em conta alguns critérios: o acesso às plataformas (é acessível? Pode usar-se em Pc e Macs?), a direção da narrativa (como é que o tema se presta a outros ambientes? Como é que a história se enquadra nos padrões educacionais?), os objetivos e as metas a alcançar ( $O$ que é que se espera que os alunos aprendam sobre eles próprios? Que competências vão desenvolver?). Os principais aspectos que devemos considerar são sempre: conhecer a audiência; delinear os objetivos; identificar as necessidades dos alunos; criar uma ligação emocional ao conteúdo e saber que não existe uma maneira certa ou errada de contar uma história transmídia.

As narrativas transmídia são hoje objeto de estudo pelos que se interessam pelas questões de inovação em educação. Espera-se que a abordagem transmídia venha a constituir uma estratégia poderosa para o ensino por causa do nível de envolvimento e profundidade que este tipo de experiências com narrativas transmídia oferece. No entanto, é necessário continuar a estudar e investigá-las para que se tornem aceitas na educação (Stackelberg, 2011; Pence, 2012).

\section{Potencialidades das Narrativas Transmídia}

Estudos realizados sobre o uso das narrativas transmídia em contexto educativo tem como objetivos compreender melhor as características da narrativa transmídia em contexto escolar e explorar os benefícios pedagógicos da sua aplicação num contexto de educação formal permitem verificar que a narrativa transmídia melhora a 
alfabetização digital e permite um envolvimento dos estudantes nas atividades escolares mais ativo e participativo do que o habitual. $O$ estudo demonstrou que a motivação intrínseca parece ter sido o ponto alto num grande número de estudantes (simplesmente pelo fato de participarem numa história real ou fictícia mais próxima dos seus interesses pessoais e sociais e de práticas semelhantes às que ocorrem fora da escola). Segundo os autores, a narrativa é uma boa ferramenta para a integração das tecnologias de informação e comunicação nos conteúdos curriculares, assim como para o desenvolvimento de práticas de alfabetização digital. Nas conclusões, sugerem que seria importante ampliar os tipos de práticas transmídia testando e experimentando outras configurações e permitindo outros tipos de interação e participação (Illera e Castells, 2012).

No Brasil, foi desenvolvido um outro projeto com o objetivo estimular o desenvolvimento da leitura e ensinar a técnica de escrita a todos os alunos da Rede Pública Estadual de Ensino. Almanaque da Rede é uma plataforma de escrita criativa e cultura digital, onde os alunos começaram por contar as histórias em papel a partir de um jogo para depois as divulgarem na Internet. $O$ que se verificou é que houve uma grande adesão às histórias o que levou os alunos a ter um maior cuidado na escrita com o objetivo sempre de melhorar e captar cada vez mais a atenção dos leitores. Verificou-se ainda que, para os mais jovens as histórias contadas em transmídia são um método poderoso para transmitir mensagens, temas e informações (Rodrigues, 2011).

Como isso se relaciona com a gamificação? As narrativas transmídia podem ser especialmente útil durante as fases de mudança de níveis ou andaimes. Leve os nossos alunos através de uma história, de preferência uma que mude com base nas escolhas que eles fizerem e como eles querem passar por isso. Dê-lhes o que eles precisam para completar partes da história. Fazendo dessa forma, quando bem feito, teremos muito mais impacto sobre eles do que se proporcionarmos o dispositivo de pontos para realizarem as atividades. O sentido de propósito que uma história pode dar é muito poderoso - mesmo se é mais uma história curta, em vez de um épico!

\section{Desenho da Investigação}

A discrepância entre a evolução da tecnologia e a pouca utilização da mesma nas salas de aula, conduziu a que o objetivo deste projeto fosse o de produzir conhecimento sobre as potencialidades das narrativas transmídia em contexto educativo e das suas implicações nas práticas pedagógicas dos professores. Pretende-se pois, contribuir para o aprofundamento de um novo conceito, visando compreender de que forma é que ele poderá permitir melhorar as práticas pedagógicas e aproximar os alunos das 
aprendizagens escolares. Como é que os professores veem e utilizam as tecnologias digitais, que tipo de atividades e situações de aprendizagem podem ser desenvolvidas para fins educativos com base no conceito de narrativas transmídia e como é que as narrativas transmídia afetam o funcionamento e a dinâmica das práticas de ensino e de aprendizagem, são as questões de investigação deste projeto de pesquisa. Dada a componente de intervenção do estudo, visando o desenho de atividades com o objetivo de melhorar, do ponto de vista metodológico, as práticas educativas, optou-se por reúne a experimentação à possibilidade de descrever, captar e analisar a interação entre pesquisa e ação em termos de complexidade e terá uma abordagem qualitativa baseada na perspectiva histórico-cultural de ensino-aprendizagem, cuja análise crítica é ancorada no pressuposto epistemológico da perspectiva sócio histórica de Vygotsky $(2001 ; 2008)$, para extrair, através da análise dos materiais didáticos e audiovisuais, as potencialidades dos recursos inseridos em um cenário contemporâneo da educação e da cultura digital no ensino de ciências. Apoiados nas características desta metodologia e tendo em conta os objetivos do projeto, desenhou-se um plano de investigação com quatro fases como podemos observar na Tabela 1 (Desenho de Investigação). A primeira fase da investigação (caracterização) visa a análise e caracterização do contexto onde a intervenção irá ter lugar, a revisão de literatura e a seleção dos participantes. Na segunda fase (criação/produção), o objetivo é trabalhar com um grupo de professores em ordem a eles próprios vivenciarem e criarem situações de aprendizagem numa perspectiva transmídia. Num primeiro momento, para os introduzir e preparar relativamente à noção e práticas transmídia e num segundo momento, já com os conhecimentos e experiências adquiridos, levar esses mesmos professores, em conjunto com o investigador, a criar e planificar atividades transmídia para os seus alunos.

No final desta fase serão selecionados três professores para na fase (observação da ação), implementarem nas suas turmas a estratégia transmídia planificada e produzida por eles. Será feita uma observação, análise e reflexão do processo e resultados obtidos por cada turma. A quarta fase de investigação é a da avaliação retroativa e reflexão sobre o processo de criação das atividades e do próprio processo de observação do funcionamento e dinâmica das práticas pedagógicas. Nas diferentes fases fazemos recurso de observação de aulas, notas de observação, análise documental (projeto educativo, planificações, programa curricular) e questionários aos professores e alunos, entrevistas semiestruturadas aos professores, registos de vídeos e fotografias, diário de bordo, conversas informais com professores, alunos e especialistas.

\section{Conclusão}


Um estudo sobre o modo como as narrativas transmídia podem ajudar a transformar as práticas educativas gamificadas, tornam-se necessárias diante da ausência de estudos nessa área no Ensino de Ciência.

Começando pela explicitação do conceito de narrativas transmídia e de uma discussão das suas potencialidades para fins educativos, concluímos o projeto com a apresentação de um esboço de investigação que pode ser desenvolvido na disciplina Química com um conjunto de professores e alunos.

A partir dessas considerações, já podemos delinear algumas situações como: as afirmações sobre narrativas transmídia e gamificação que são feitas, muitas vezes no senso comum, encontram respaldo em pesquisas acadêmicas. Os métodos transmissivos de ensino, praticados pela maioria das instituições escolares, não são mais capazes, por si só, de atender as demandas de indivíduos que incorporam cada vez mais as características da cultura digital, como o fácil acesso à informação através das tecnologias digitais, entre outras características. Esses modelos empiristas de ensino, que se baseiam na premissa de que o conhecimento deve partir do professor para os alunos, e a estes cabem apenas o comportamento passivo de receber o que vier do professor, estão sendo questionados e revisados, ao menos no âmbito acadêmico. Espera-se também que sejam revisados e questionados fora da academia, nos ambientes de aprendizagem. Assim, a gamificação surge como importante ferramenta prática no sentido de abrir mais algumas brechas nesses modelos.

\section{Referências}

Bottone, J. (2012). Transmedia Design for Education and Training. In The Interservice/Industry Training, Simulation \& Education Conference (I/ITSEC). Volume: 2012. Orlando, FL. Disponível em: http://www.iitsec.org/attendees/Documents/11Abstracts.pdf

Costa, F. (2007). Tendências e práticas de investigação na área das tecnologias em educação em Portugal. In A. Estrela (Org), Investigação em educação: Teorias e práticas (1960-2005) (pp.169-224). Lisboa: EDUCA | Unidade de I\&D de Ciências da Educação.

Fleming, L. (2011). A New Model of Storytelling: Transmedia. Disponível em http://www.edutopia.org/blog/transmedia-digital-media-storytelling-laura-fleming 
Baltic Film and Media School Screen Studies. Tallinn: Tallinn University Press

Illera, R., \& Castells, N. (2012). Usos educativos de la narrativa transmedia. La Carta Ancestral Universidade de Barcelona.

Gomez, J. (2011) Globo News - saiba mais sobre o diálogo coletivo chamado transmídia. In The Alchemists. Disponível em: http://www.thealchemists.com/blog/video s/globo-news-saiba-mais-sobre-odi\%C3\%A1logo-coletivo-chamado-transm\%C3\%ADdia/

Jenkins, H. (2003). Transmedia storytelling. Moving characters from books to films to video games can make them stronger and more compelling. In MIT Technology Review. Disponível em: http://www.technologyreview.com/news/401760/transmediastorytelling/page/3/

Jenkins, H. (2009). Cultura da convergência: a colisão entre os velhos e novos meios de comunicação. São Paulo: Aleph.

Kinder, M. (1993). Playing with Power in Movies, Television, and Video Games: From Muppet Babies toTeenage Mutant Ninja Turtles. Berkeley: University of California Press.

Long, G. (2009). Transmídia: a narrativa da atualidade. In Revistapontocom. Disponível em: http://www.revistapontocom.org.br/edicoes-anterioresentrevistas/transmidia-a-narrativa-daatualidade

NG, C. (2012). Transmedia Literacy and Digital Storytelling in Education. Disponível em:http://www.slideshare.net/fullscreen/ngcharmaine/transmedia-storytelling-and-digitalliteracy ineducation-13202271/2

Pence, H. (2012). Teaching with Transmedia. In Journal of Educational Technology Systems. Issue: Volume 40, Number 2/2011 (pp. 131-140). State University of New York at Oneonta.

Porto-renó, D., Versuti, A. C., Moraes-Gonçalves, E., \& Gosciola, V. (2011). Narrativas transmídia: diversidade social, discursiva e comunicacional, 14 (2), 201-215.

Pratten, R. (2011). Getting Started in Transmedia Storytelling: A Practical Guide for Beginners. Seattle: CreateSpace. Project Tommorow. (2012). Personalizing the Classroom Experience - Teachers Librarians and Administrators Connect the Dots with 
Digital Learning.

Rodrigues, S. (2011, 8/11/2011). Sónia Rodrigues e o projeto Almanaque da Rede. In Conexão do Professor: tudo que interessa ao professor da rede estadual. Acesso em

http://www.conexaoprofessor.rj.gov.br/especial.asp?EditeCodigoDaPagina=1956

Scolari, C. (2009). Transmedia Storytelling: Implicit Consumers, Narrative Worlds, and Branding in Contemporary Media Production - International Journal of Communication 3 (pp. 586-606).

Stackelberg, P. (2011). Creating Transmedia Narratives: The Structure and Design of Stories Told Across Multiple Media. A Master's Thesis Presented to School of Information Design and Technology State University of New York Institute of Technology The Design-Based Research Collective. (2003). Design-Based Research: An Emerging Paradigm for Educational Inquiry. Educational Researcher, 32(1), 5-8.

Vygotsky, L.S. Pensamento e Linguagem. São Paulo: Martins Fontes, 2001. 175p.

Vygotsky, L.S. A formação Social da Mente. O desenvolvimento dos processos psicológicos superiores. São Paulo: Matins Fontes, 2008. 182p. 